\title{
An Approach to Screen COVID-19 patients: With special reference to Pakistan
}

Faiza Gul Durrani ** and Farhat Zaheer*

\section{Summary}

The World Health Organisation declared Covid-19 as a pandemic on $11^{\text {th }}$ March 2020. The main approach to tackle Covid-19 worldwide is to screen and provide supportive care to patients. For screening purpose RT-qPCR based test are used as an initial detections assay. The test is expensive, time consuming and not suitable for mass scale screening/ confirmation requirement. A recent advancement is development of Immunoassay procedures (liquid Phase tests or bed side 10-20minute strip test). In order to help and accelerate bringing life to normal after lock down, Pakistan is in dire need to develop and adopt the immunoassay procedures for mass scale screening and confirmation of COVID-19 infection. It is cheap and easy to perform without a lab requirement.

\section{Introduction}

An outbreak of novel Coronavirus infection (COVID-19) in Wuhan (China), has rapidly spread throughout the world. A current estimate indicates total number of incidences of infected persons in the world is $1,452,378$, total deaths 83,614 and recovered persons are 282,147 (8thApril, 2020) ${ }^{1}$. First COVID-19 patient reported in Pakistan was on $26^{\text {th }}$ February 2020 and number of incidences reported on $8^{\text {th }}$ April 2020 was 4,196 cases, deaths $60(1.42 \%)$ and the number of recovered cases, 467(11.1\%) According to the Pakistan ministry of health report, the projected number of incidences is 50,000 by the end of April. ${ }^{2}$

\section{Background}

Since 1960s, Coronaviruses have been recognized as one of the infection sources responsible for common cold among humans. Six species of human coronavirus have been identified to date, (HKU1 ,229E, NL63 ,OC43,MERS and SARS-CoV). The pandemic COVID-19 virus has been designated as a sister to SARS-CoVs, hence the name SARS-CoV-2 by a coronavirus study group of the International Committee on Taxonomy of viruses ${ }^{3}$. The coronaviruses are enveloped viruses with a positive-sense, single stranded RNA. The first and complete genome sequence of COVID-19 (GenBank: MN908947.3) and the structure of the main protease of virus (DOI: 10.2210/pdb6lu7/pdb) have been reported ${ }^{4}$. Its genome constitutes 29891 nucleotides, encoding 9860 amino acids. The virus has 70 percent similarity in genetic sequence with SARS. The X-ray crystallographic protein data bank structure explained the glycosylated crown shape covering (hence the name "corona", Latin for crown). These spike proteins facilitate the virus to bind to the receptors on the cells. The covid-19 has two $\mathrm{S}$ domains - S1 and S2. The computational analysis of S1 and S2 has shown the importance of the S1 domain which has many unique residues. These not only bind to CD26 receptor cells but also ACE2 cells ${ }^{5}$. It has been reported that this particular virus can infect human respiratory epithelial cells 1001000 times more as compared to previous corona strains because of its unique binding sites for ACE2 receptors. In severe cases, it can cause organ failure and even death ${ }^{6}$.

For a viral infection/medical illness there is a set route of diagnosis and treatment. The strategy advised by WHO is based on screening and providing supportive system to the patients in need. Throughout the world, screening of the infected individuals is essentially based upon: thermal monitoring of the body temperature, breathing problem and dry cough. It is noteworthy to mention here that clinical symptoms 
after infection would appear after a period of 3-5 days of infection. These may be delayed for two weeks, besides the fact that some of the infected subjects may remain asymptomatic ${ }^{7}$. Infected individuals identified by screening are isolated at least for 14 days (quarantine) as per WHO guidelines.

\section{Diagnostic tests for COVID-19 with special reference to Pakistan}

In Pakistan, at present in order to confirm the COVID-19 infection, only molecular based RT- qPCR is allowed. RT-PCR technique is very well established in all the research institutes of the country ${ }^{17}$. In particular case of COVID-19, It is performed with the kits available to the Govt. (imported purchase or donation). Most of the kits contain 3 assays in which the targets are Orf1 gene ( human RNA polymerase protein) E-gene (envelope protein) and the $\mathrm{N}$-gene ( the nucleocapsid protein $)^{8}$ so if the virus does mutate the chance of all three targets mutation is low, however if the results of 1 or 2 of these essays is not positive then the result needs to be revised. A possibility of a false negative and a false positive cannot be excluded and in order to confirm the results it needs to be repeated for verification. Bearing in mind that the procedure is lengthy, requires BSL-2 Lab facility and it is an expensive test especially in view of repeat required ${ }^{9}$. In patients with confirmed COVID-19 diagnosis, the laboratory evaluation should be repeated to evaluate for viral clearance prior to being released from observation.

The possibility of a local RT-q PCR kit production is being explored or it is in the process of validation in Pakistan. However, the primers are not synthesised in Pakistan. Though, at present the requirement of tests is being managed by the country adequately as far as possible however it is still limited to a single test performance, with hardly required repeat tests as mentioned above. Also, in view of the rising number of infected patients being identified, the country will reach an exhaustive stage on how to manage the required number of tests. It will not be possible to manage the required lab workload; the country requires to be prepared for that situation.

The diagnostic test in particular for COVID-19, approved by WHO in its briefing (early March, 2020) was by RT-qPCR. However, WHO also emphasized the importance of immunoassay serological tests, which can be used as bedside tests and will be a big breakthrough for testing service at mass level. These immunoassay-based tests reagents/kits have been manufactured by several countries including USA, China, South Korea, Ireland etc. ${ }^{12,13}$. WHO is working on its validation. As soon as the validation is completed, these tests will be recommended because of their specificity for detection of antibody $\mathrm{IgM}, \mathrm{IgG}$ in patients who are in recovering phase or have had been previously infected,10. Moreover, the immunoassay test can also be applied for the detection of antigen (virus), during the first week of infection.

The detection of antibodies, (IgM and $\operatorname{IgG}$ ) in blood by immunochromatography procedure is simple and straightforward. These two substances are produced in blood after infection, usually by day 7 and 14 respectively. It requires a nitrocellulose strip with a sample pad, and coated anti IgM and IgG pads. To the sample pad a drop of sample (blood) is added by a finger-prick followed by buffer. The sample and buffer move laterally towards first IgM- antibody pad band where $\operatorname{IgM}$, if present in the sample will bind to antibody and a colour band is produced. Similarly, the next pad with anti-IgG coated will identify the presence of IgG produced in blood as a result of infection. Both IgM and IgG identify the status of infection - present or past respectively. A quality control colour band will also be produced at its respective position on the strip to confirm that the test procedure is working accurately. ${ }^{11}$

The immunoassay technique reagents are available both for Enzyme Linked Immunosorbent Assay (ELISA) procedure in liquid phase and Lateral Flow Immunoassay (LFIA) for immunochromatographic method. Bulk reagents, viz. antibody, active site fragments of viral protein antigen, Enzyme linked tracer for ELISA are available besides the availability of nanoparticle linked, fluorescent probes etc. The immunoassay kits for the detection of viral antigen and antibody are available internationally. ${ }^{12,13}$ As described above several countries, including China, South Korea, UK, Europe, and the USA are in process of producing immunoassay kits for COVID-19 antigen/antibody detection. The main 
consideration in hand in Western countries is the validation of the assay which is essential for quality control. It is anticipated that assays validation will be achieved in a few weeks' time if not days. Once it is achieved, there is no reason why Immunoassay technique will not become a front-line technique and priority of choice to be applied for mass scale screening and for confirmation.

A comparison of the two techniques, RT-qPCR and the immunoassay techniques are clearly indicative that immunoassay procedure is less expensive, easy to perform and results are available within 10-20 minutes ${ }^{14}$.

\section{Conclusion}

It is concluded, that Pakistan should adapt and validate the immunoassay procedures. This would be first step forward towards normalization after lockdown and quarantine situation. It will be helpful to bring the healthy recovered persons back to work, hence normalise routines ${ }^{14}$ Furthermore, Pakistan has the capability and expertise to produce immunoassay-based kits as previously developed by INMOL Lahore (PAEC) for hormones and Tumour marker assays ${ }^{15,16}$. Pakistan should invest to prepare the antibody-based immunoassay kit because of its high specificity, low in cost and easy to use methodology.

\section{REFERENCES}

1." Coronavirus Resource Centre" coronavirus.jhu.edu, viewed on $8^{\text {th }}$ April 2020, $<$ https://coronavirus.jhu.edu/>

2. Ashfaq, A "Coronavirus: More than 50,000 COVID-19 patients expected in Pakistan by end of April", Gulfnews.com, $5^{\text {th }}$ April 2020

<https://gulfnews.com/world/asia/pakistan/coronavirus-more-than-50000-covid-19patients-expected-in-pakistan-by-end-of-april-1.70815880>

3. Xu, X, Chen, P, Wang, J, et al. 2020 "Evolution of the novel coronavirus from the ongoing Wuhan outbreak and modelling of its spike protein for risk of human transmission" Science China Life Sciences; 2020;63(3):457-460.

4.Vankadari, N, Wilce, J, 2020 "Emerging WuHan (COVID-19) coronavirus: glycan shield and structure prediction of spike glycoprotein and its interaction with human CD26" Emerging Microbes \& Infections, 2020 9:1, 601-604, DOI: 10.1080/22221751.2020.1739565

5. Lu, H,Stratton, C, Tang, Y, 2020 "Outbreak of pneumonia of unknown etiology in Wuhan China : the mystery and the miracle" Journal of Medical Virology 2020;92(4):401-402.

6. Huang, C, Wang, Y, Li, X, et al. 2020 "Clinical features of patients infected with 2019 novel coronavirus in Wuhan, China" Lancet 2020;395(10223):497-506.

7.Xiaolong, Cai. 2020. "An Insight of comparison between COVID-19 (2019-nCoV disease) and SARS in pathology and pathogenesis". 10.31219/osf.io/hw34x. 
8. Broughton, J, Deng, X, Yu, G, Fasching, C, Singh et al. 2020. "Rapid Detection of 2019 Novel Coronavirus SARS-CoV-2 Using a CRISPR-based DETECTR Lateral Flow Assay" https://doi.org/10.1101/2020.03.06.20032334

9.Souf, S, 2016 "Recent advances in diagnostic testing for viral infections",Bioscience Horizons: The International Journal of Student Research, Volume 9, 2016, hzw010, https://doi.org/10.1093/biohorizons/hzw010

10. "Coronavirus disease (Covid-19) Pandemic", who.int, viewed on 1-8 April 2020, < https://www.who.int/emergencies/diseases/novel-coronavirus-2019>

11. Li, Z, Yi,Y, Luo, $X$, Xiong, N, Liu, Y et al. 2020. "Development and Clinical Application of $A$ Rapid IgM-IgG Combined Antibody Test for SARS-CoV-2 Infection Diagnosis" Journal of Medical Virology. 10.1002/jmv.25727.

12. "coronavirus ELISA kits/Biocompare", viewed on 1-8 April 2020 $<$ www.biocompare.com $>$

13. "coronavirus COVID-19 IgM ELISA Assay kit-Eagle Bioscience" viewed on 1-8 April 2020 $<$ www.eaglebio.com $>$

14. "Expert reactions to Coronavirus", sciencemediacentre.org, viewed on 1-8 April 2020 <https://www.sciencemediacentre.org/tag/covid-19/>

15. Zaheer, F. 1992 "Behaviour of 1-125 Labelled Monoclonal anti TSH, and Cellulose Linked (Solid Phase) antibody in a supersensitive TSH IRMA "IAEA-SM324/69: 73-81. proc. on Dev. RIA and related procedures Vienna Austria.

16. Zaheer, F. 1992 "New perspectives for Radioimmunoassay in developing countries "IAEA-SM-324/71: 579-583. proc. on Dev. RIA and related procedures Vienna Austria.

17. Durrani FG, Gul R, Sadaf S, Akhtar MW.2015 "Expression and rapid purification of recombinant biologically active ovine growth hormone with DsbA targeting to Escherichia coli inner membrane". Applied Microbiology and Biotechnology. 99(16):6791-6801. DOI: 10.1007/s00253-015-6751-6.

*Author: Farhat Zaheer (TI) Ph.D.)

Retired: Chief Scientific officer (CSO) Institute of Nuclear Medicine and Oncology Lahore (INMOLPAEC), Currently Resident in London UK.

Email: farhatzaheer@hotmail.com

Co-author**: Faiza Gul Durrani (Ph.D.)

Consultant: (Science shines Ltd) London.

Email: faizaguldurrani@gmail.com 
